\title{
PARTICIPACIÓN EN COMUNIDADES DE APRENDIZAJE, COMO ELEMENTO CLAVE PARA LA FORMACIÓN INICIAL EN EL ÁMBITO DE LA EDUCACIÓN INCLUSIVA E INTERCULTURAL
}

\section{PARTICIPATION IN COMMUNITIES OF LEARNING AS A KEY ELEMENT FOR INITIAL TRAINING IN THE FIELD OF INCLUSIVE AND INTERCULTURALEDUCATION}

\author{
Martha Orozco Gómez ${ }^{1}$. Universidad de Burgos. España. \\ mlorozco@ubu.es
}

Sergio Sánchez Fuentes. Universidad de Burgos. España.

José Luis Cuesta Gomez. Universidad de Burgos. España.

Asunción Cifuentes García. Universidad de Burgos. España.

Rosario Ángela Martín Almaraz. C.E.E. Nuestra Señora de la Esperanza. Universidad Rey Juan Carlos de Segovia, España.

\section{RESUMEN}

En este artículo se describe y analiza la participación de los futuros maestros, en la actualidad estudiantes de Grado de Magisterio de la Universidad de Burgos, como voluntarios en una Comunidad de Aprendizaje, que, al igual que las demás, por su concepción de la educación y la organización y metodología en que se plasma, ofrece una plataforma privilegiada para la aproximación a la práctica, desde la Universidad. Igualmente muestra cómo esta participación contribuye al desarrollo de un ámbito formativo fundamental, constituido por los valores y actitudes, que en los actuales Planes de Estudio del Grado de Primaria están vinculados a las competencias transversales. Los autores concluyen que existe una necesidad de coordinar, colaborar y realizar feedback entre los centros escolares y la propia universidad en la formación que se otorga a los futuros maestros y maestras en nuestro país. Además, se explicita la oportunidad que las comunidades de aprendizaje están dando para una formación de calidad a los alumnos en las aulas educativas.

PALABRAS CLAVE: comunidades de aprendizaje, interculturalidad, inclusión, educación, universidad, voluntariado, equidad.

\section{ABSTRACT}

This article describes and analyzes the participation of future teachers, currently undergraduate students of Education of the University of Burgos, volunteering at a Learning Community, which, like the others, for their conception of education and the

\footnotetext{
${ }^{1}$ Martha Orozco Gómez: Profesora Ayudante Doctora en la Universidad de Burgos, Departamento de Ciencias de la Educación. Burgos, España.
} 
organization and methodology that is plasma, offers a unique platform for the approach to practice, from the University. Also it shows how this participation helps develop a basic training area, consisting of the values and attitudes, which in the current Curriculum Grade Primary are linked to generic skills. The authors conclude that there is a need to coordinate, collaborate and feedback between schools and the university itself in the training of future teachers is given in our country. In addition, the opportunity explicit learning communities are paying for quality training to students in educational classrooms.

KEY WORDS: learning communities, inclusión, education, university, volunteering, equity.

\section{Cómo citar el artículo:}

Orozco Gómez, M., et al. (2016). Participación en comunidades de aprendizaje, como elemento clave para la formación inicial en el ámbito de la educación inclusiva e intercultural. Revista de Ciencias de la Comunicación e Información, 21(1), 35-44. doi: http://doi.org/10.35742/rcci.2016.21(1).35-44

\section{INTRODUCCIÓN}

Sin duda alguna, la realidad va superando las previsiones que tenemos sobre los diferentes ámbitos de la vida en la cual nos movemos y que determinan nuestro día a día. En este sentido, nos hemos percatado de la necesidad de formar a los nuevos maestros en los temas relacionados con las desigualdades e injusticias propias del sistema que rige el mundo actual y también con la interculturalidad y el desarrollo humano sostenible que deseamos. En otras palabras, aquellos temas que nos hacen más sensibles a los problemas y necesidades de los demás seres humanos, que nos aportan herramientas para aprender a convivir mejor, a compartir, en definitiva a contribuir a hacer un mundo mejor y más sostenible para todos. Sin embargo, cada vez se ha ido haciendo más evidente el desfase entre los programas universitarios, dirigidos a la formación de maestros y la realidad del mundo que nos rodea y más concretamente de la escuela, su contexto y la creciente complejidad en que se mueve. Esta evidencia es la que ha permitido que en los programas propios de los actuales Planes de Estudio de Grado ya se pueda contar con asignaturas concretas que trabajan en esta dirección, sin olvidar que los maestros que se encuentran en ejercicio actualmente sufren la carencia de este tipo de contenidos en su formación inicial y se ven obligados en muchos casos a improvisar, o bien movidos por su preocupación o compromiso a buscar alternativas a estas carencias en seminarios y cursos especiales, en los cuales se va analizando la propia dinámica que la sociedad va imprimiendo a la educación.

\subsection{Importancia del desarrollo de actitudes en la formación inicial}

Un avance importante es que hoy en día podemos hablar de la existencia de un consenso claro entre los autores respecto a los elementos clave de la formación inicial de maestros en ámbitos como el de la interculturalidad, uno de los principales que plantea respuestas a esta complejidad y diversidad del mundo en que vivimos. 
Varios de ellos, subrayan la importancia de que se perciban con sensibilidad y apertura la existencia de la diversidad en la escuela y se muestren interesados por indagar en los principios de la interculturalidad, centrados en el valor del respeto a la diferencia (Merino y Leiva, 2007) y por su aplicación a la práctica. Otras autoras (Aguado, Gil y Mata, 2007), afirman que esta formación inicial debe ayudar al futuro profesor o maestro a comprender que vive en una sociedad diversa y debe implicarle en un proceso de reflexión crítica, que le ayude a desvelar sus suposiciones o prejuicios ocultos y sobre todo a irlos eliminando progresivamente, para caminar hacia actitudes positivas.

Con el ánimo de señalar antecedentes en esta línea, consideramos importante recoger alguna propuesta de formación inicial, anterior a los actuales planes de estudio de Grado, como la de las autoras Cabrera, Espín, Marín y Rodríguez (1999) que ya apuntaban hacia el desarrollo de competencias, incidiendo también en esta formación en actitudes y valores, especificada en forma de pretensiones del modo siguiente:

- Percibir la diversidad desde la diferencia, no desde el déficit,

- Experimentar y vivenciar en contextos interculturales.

- Desarrollar una actitud favorable a la diversidad cultural

- Reflexionar sobre el currículum oculto, del profesorado y el modo en que puede afectar a su trabajo.

- Aceptar los valores inherentes a la educación intercultural y comprometerse con ellos

Sin embargo, una vez implantados los nuevos planes de Grado, la interculturalidad no está tan presente como era de esperar y desear, pues la mayoría de las asignaturas de dichos planes no reflejan apenas la realidad multicultural de nuestro país, tal como apunta algún estudio (Goenechea, 2008). El estudio publicado por esta autora muestra que más de un50\% de los actuales planes de estudio de Magisterio, Educación Social, Pedagogía y Psicopedagogía no tienen asignaturas sobre diversidad cultural y los que las tienen la sitúan en el espacio de la optatividad.

\section{OBJETIVOS}

El objetivo principal de este trabajo es describir una experiencia que pretende ser la aproximación a la realidad y el desarrollo de actitudes, a partir de una asignatura en la Facultad de Ciencias de la Educación, de la Universidad de Burgos. No obstante, hay asignaturas que permiten incidir de forma especial en este desarrollo de actitudes como la Educación para la paz y la igualdad -asignatura que se encuentra dentro del plan de estudios del primer año de Educación Infantil y Primaria-, dentro de la cual se trabajan módulos como interculturalidad o cooperación al desarrollo y que ha permitido crear un vínculo real entre los centros educativos y la comunidad a través de las ONG que se dedican prioritariamente a estos temas. Actividades tales como realizar programaciones con el asesoramiento de estas entidades y posteriormente aplicarlas en centros de Educación Infantil y Primaria. Esta práctica que se ha llevado a cabo con estudiantes de primer curso, ha sido una experiencia inolvidable para todos, creando conciencia de la realidad, conduciendo a un conocimiento de todo el contexto de la ciudad en que estudian y 
lo que está ocurriendo en ella: sus movimientos, sus avances, sus retrocesos... y sobre todo propiciando esa primera aproximación a lo que será su espacio profesional con la ilusión de poder aportar un granito de arena para un mundo mejor.

Sin duda todo ello ha supuesto un buen comienzo para el largo recorrido que debemos hacer todos juntos. Cabe reseñar que las experiencias que se han ido teniendo en estos últimos cursos a través del planteamiento de esta asignatura son realmente significativas, tanto para los maestros/as en proceso de formación, como para quienes intervinimos en ese proceso, pues sin duda alguna es fundamental la actitud y compromiso de quien forma, teniendo en cuenta que este planteamiento requiere tomar un poco de distancia con respecto al aula de clase y esto significa emplear dosis adicionales de dedicación, esfuerzo y tiempo-. El contacto directo con la realidad, ver lo que sucede alrededor, teniendo como referente teórico unos contenidos predeterminados en una asignatura hace que el/la estudiante sea realmente consciente de cuál va a ser su papel, las dificultades con las que se puede encontrar y el tipo de sociedad en que nos encontramos inmersos. Los estudiantes participantes en estas actividades de contacto con la realidad también han tenido la oportunidad de intervenir en tertulias dialógicas con los estudiantes de la Universidad de la Experiencia, tanto jóvenes como mayores y han evaluado muy positivamente ese encuentro intergeneracional, que supone aprender más allá de la lectura que nos ha servido como "excusa" para estos encuentros inolvidables.

\subsection{Las Comunidades de Aprendizaje y sus potencialidades para el desarrollo de actitudes en el marco de la educación inclusiva}

También se ha intentado fomentar este vínculo entre los centros de formación y la realidad, a través de la participación en experiencias innovadoras, que ya se están implantando en numerosas localidades españolas, como las Comunidades de Aprendizaje que consisten, según sus creadores, en un Proyecto de transformación social y cultural de un centro educativo y de su entorno para conseguir una Sociedad de la Información para todas las personas, basada en el aprendizaje dialógico, mediante la educación participativa de la comunidad, que se concreta en todos sus espacios, incluida el aula. (Aiguadé, Saso, Gallart y Carol, 2003).

Es evidente que la característica más clara que comparten las Comunidades y las experiencias en que se apoyan (Éxito escolar para todos/as, Programa de desarrollo Escolar y Escuelas aceleradas) es su carácter inclusivo, que se manifiesta en el deseo firme de transformar la escuela, para responder a las necesidades de todos los alumnos, independientemente del medio en que se encuentre, por más desfavorecido que sea. Conviene ahora destacar, de cara a los objetivos que nos planteamos en este trabajo que los contextos donde estas experiencias se han organizado y siguen desarrollándose son multiculturales. Actualmente, cuando ya se encuentran muy extendidas, pues superan el centenar, estamos en condiciones de constatar que la mayoría de las Comunidades se han constituido en verdaderos centros donde coexisten diversas culturas, ofreciendo por tanto una plataforma privilegiada para desarrollar los valores propios de la educación intercultural. Existen elementos clave en las Comunidades, que son decisivos para desarrollar estos valores, como el aprendizaje dialógico, que se 
traduce a la práctica en la metodología de los grupos interactivos, consistentes en la introducción en el aula de las interacciones necesarias para que todo el alumnado pueda seguir los contenidos y realizar las actividades, sin quedarse rezagados respecto a los compañeros. Esto se consigue, por una parte, mediante la incorporación de más personas adultas al aula, sean padres y madres, otros familiares, profesores, voluntarios como los estudiantes de magisterio entre otros... y por otra, mediante el establecimiento de pequeños grupos. El aula se divide en cuatro grupos de alumnos heterogéneos en cuanto a nivel de aprendizaje, contexto sociocultural y género y las personas adultas se encargan de dinamizar cada uno de estos grupos, durante el período de tiempo en que se realiza la actividad, procurando favorecer las interacciones entre estos alumnos y la colaboración entre ellos. La profesora tutora se encarga de coordinar todos los grupos, así como de asegurar la coherencia de las actividades que todos ellos, de forma rotatoria, van realizando.

En algunas investigaciones realizadas (Foncillas, Petreñas, y Cifuentes, 2012) se ha comprobado que estos grupos suponen una auténtica alternativa a la separación por niveles en las aulas, ya que los resultados de aprendizaje en los alumnos que participan son muy diferentes, a favor del primer tipo de agrupamiento, es decir que es el camino más eficaz para alcanzar el éxito escolar para todo el alumnado. Hay que tener en cuenta que la incorporación de más adultos al aula, así como la interacción entre alumnos que emplean como vehículo para comunicarse un lenguaje más próximo al de los compañeros que el que emplea el profesor, son garantía de eficacia.

Pero además de su potencial inclusivo, queremos subrayar la importancia que esta relación estrecha o interacción entre alumnos tiene para el diálogo y el respeto intercultural, ya que se establece organizando grupos heterogéneos, donde no solo se sientan juntos los alumnos de diversas culturas, sino que conviven auténticamente, compartiendo de forma natural la apasionante experiencia de aprender. $Y$ finalmente en esta forma de organizar el aula y los grupos se fomenta la solidaridad y el saber escuchar y respetar a los demás, lo cual repercute lógicamente en el clima de las clases. Una vez enunciadas algunas de las líneas generales del trabajo de las comunidades de aprendizaje, queremos dedicar los siguientes apartados a presentar la experiencia de colaboración entre el alumnado de la Facultad de Humanidades y Educación de la Universidad de Burgos con una Comunidad de aprendizaje de la ciudad. Esta experiencia ya tiene un recorrido de seis años y creemos que está dando frutos importantes, tanto de cara al centro donde se realiza, como de cara a la formación de los alumnos de las diplomaturas de magisterio y licenciatura en Pedagogía en su comienzo y actualmente el de los grados de Infantil y Primaria. A pesar de que aún no se ha realizado un estudio sistemático de las aportaciones de esta experiencia, tanto las entrevistas orales o escritas con los alumnos participantes, como la observación y reflexión realizada por la coordinadora del trabajo de los alumnos voluntarios, avalan esta consideración.

\section{METODOLOGÍA}

Las experiencias de aproximación a la realidad que se han ido realizando desde las universidades no tendría sentido sin la implicación de la escuela, es así como 
podemos incluir en este compartir de vivencias la llevada a cabo en el Colegio "Apóstol San Pablo" un centro privado concertado creado en el año 1975 y transformado en Comunidad de aprendizaje en 2005. Actualmente en este Centro se imparte Educación Infantil y Educación Primaria. Es un centro pequeño, acogedor y familiar y en el que todos pretenden ser una gran familia: profesores, padres, alumnos, personal de servicios, voluntarios y todas las demás personas que de alguna forma tienen relación con él. Durante cuatro años, el Claustro de Profesores, desarrolló un Proyecto de interculturalidad. Fruto del trabajo y estudio de este proyecto se ha creado un nuevo Proyecto Educativo del Centro, Proyecto Curricular y un Plan de Acogida. Estos proyectos sitúan en su núcleo la educación intercultural, entendida de forma integral, valorando la gran riqueza que supone para el conjunto del alumnado. La diversidad de culturas existentes en el Centro es lo que ha motivado e ilusionado a la comunidad educativa para intentar crear un modelo de educación que respete y valore todo lo positivo que pueden aportar el diálogo entre las mismas. Como fruto de este Proyecto y de su puesta en práctica, el clima que se respira actualmente en las aulas es de un gran respeto a la diversidad existente en las mismas. Posteriormente, en el año 2005 el profesorado con el apoyo de las familias, realizó su sueño y transformó el centro en Comunidad de Aprendizaje, cuyas características principales e han expuesto en las páginas anteriores. Una vez transformado en Comunidad del voluntariado se ha revelado como elemento clave en los grupos interactivos que se han puesto en marcha, puesto que contribuye a aumentar, tanto el número de interacciones que viven los niños, como la calidad de estas interacciones en un plano de diálogo igualitario, influyendo así poderosamente en la mejora de su aprendizaje.

Tal como se concibe el voluntariado en las comunidades, cualquier persona adulta puede estar dentro de la clase, aunque esto no signifique en ningún caso que el maestro o maestra pierda su rol dentro del aula; sino que mientras los voluntarios vienen a ayudar y a potenciar las interacciones, el tutor del grupo continúa siendo quien gestiona y organiza. Esto supone que en ningún caso, el voluntario está solo en el aula, sustituyendo las funciones propias del maestro o maestra.

En este centro, el voluntariado ha ido cambiando con el tiempo, como resultado de la difusión de los Proyectos de Interculturalidad y Comunidades que ha tenido siempre, entre otras, esa intencionalidad. Cuando se comenzó con este Proyecto el colegio tenía jornada de mañana y tarde, por lo cual, en los primeros años se contaba con un buen número de estudiantes de Magisterio de la Universidad, que estudiaban por las mañanas y asistían alguna tarde a colaborar con el profesorado. También al principio se contaba con un reducido número de familiares, trabajadores del Centro Cívico del barrio y algunas personas de distintas asociaciones de voluntariado que podían venir por las tardes.

A partir del curso 2011-12, el colegio modificó la jornada y se pasó a tener jornada continua por lo cual, se produjeron algunos problemas ya que al tener jornada de mañana, el número de voluntarios, en un principio, se redujo bastante. Muchos estudiantes de Magisterio estudiaban por las mañanas y su horario era coincidente con el Centro, por lo cual no podían acudir a él. Por otra parte, algunos tenían las mañanas de los viernes libres y venían a colaborar voluntarios pero era un número bastante elevado y hubo que organizar en casi todos los cursos los grupos interactivos ese día para poderlos ubicar a todos. 
Es importante resaltar la relevancia que ha tenido y tiene para esta Comunidad de aprendizaje el grupo de alumnos de prácticas, pues suponen una gran ayuda para el centro que, a su vez, contribuye poderosamente a su formación, ya que el propio programa formativo del Prácticum se encamina a la relación teoría-práctica y a la inmersión reflexiva en la vida del centro, conociendo desde dentro los aspectos más importantes del mismo, tales como la labor tutorial y la metodología de los profesores (en este caso basada en el aprendizaje dialógico) y muy especialmente el modo en que se establecen las relaciones con las familias de los alumnos y otras personas que forman parte del contexto que rodea el centro. Algunos alumnos, después de terminar sus prácticas se incorporan al voluntariado, porque les resulta realmente motivador y significativo el Proyecto de Comunidades de aprendizaje.

Considerando que el voluntariado exige un compromiso de continuidad, seriedad y responsabilidad hacia el Proyecto, se ha establecido un protocolo o ficha para formalizarlo y firmarlo al comienzo de curso, por parte de cada persona que se presta al voluntariado, con la intención de que se mantenga a lo largo de dicho el curso, salvo que existan alguna causa justificadas para su interrupción. El pasado curso se creó una comisión de voluntariado, encargada de gestionar la incorporación de personas voluntarias a las diferentes actividades del centro y el aula, así como de preparar un plan de formación, centrado en las cuestiones fundamentales que se consideran básicas para colaborar eficazmente en una Comunidad de Aprendizaje (Características de las comunidades de aprendizaje, líneas generales del aprendizaje dialógico, los grupos interactivas y sus potencialidades, el funcionamiento de los grupos interactivos y el papel de los voluntarios como dinamizadores de los grupos interactivos).

Una vez realizado este periodo formativo, los voluntarios colaboran fundamentalmente en los grupos interactivos que, tal como expusimos anteriormente, son una forma flexible de organizar el trabajo educativo en el aula. Insistimos en la importancia de esta metodología pues con ella se consigue favorecer la interacción entre iguales y activar el trabajo en equipo, ya que se trata de llegar a un objetivo común, a partir de las aportaciones de cada uno de sus miembros.

Para trabajar en los grupos se les propone una guía de observación-reflexión que deben realizar cada día y en la cual deben recoger los siguientes aspectos:

a) Una descripción de la tarea o actividad que realizan los alumnos, estimando su grado de dificultad.

b) Una descripción del modo en que los niños se ayudan entre sí.

c) Una descripción del modo en que se ha dinamizado el grupo, para facilitar el diálogo y la ayuda mutua entre sus miembros

Finalmente, al terminar su periodo de voluntariado se les pide que realicen por escrito una evaluación-reflexión sobre los grupos interactivos y el Colegio en general, que posteriormente se comparte con los compañeros y el profesorado del Centro. Así mismo, los voluntarios realizan tareas de apoyo al alumnado con necesidades educativas especiales o con dificultades de aprendizaje dentro de las aulas, en coordinación estrecha con los tutores correspondientes, haciendo posible este principio propio de la educación inclusiva, que se expresa diciendo que los 
alumnos forman auténticamente parte del grupo-clase. Para ello dispondrán de los recursos necesarios dentro del aula sin tener que salir de ella. De este modo se evita al establecimiento de agrupamientos por niveles, que acaban consolidándose como tales y tras segregar al alumnado, no conducen al éxito en el aprendizaje. Recogemos en esta última parte de nuestro trabajo los párrafos que condensan las reflexiones de dos alumnos-voluntarios, tras su participación en los grupos interactivos en el Centro del que venimos hablando, que se completan posteriormente con las de los alumnos de $5^{\circ}$ y $6^{\circ}$ curso del mismo y de la profesoratutora, coordinadora del voluntariado.

\section{RESULTADOS}

Reflexión de dos voluntarios, alumnos del grado de Primaria de la Facultad de Humanidades y educación de Burgos:

- "La experiencia es muy positiva. Me ha ayudado a hacerme una idea veraz del funcionamiento de un aula y a ponerme en contacto directamente con los niños desde una perspectiva alumno-docente. En un primer momento me dominaba la inseguridad de no saber dirigir las actividades o de no poder ayudar a los niños lo mejor posible, aunque ya sabíamos que nuestra función no era la de aportar contenido, sino la de dinamizar el funcionamiento del grupo. Algo que me ayudó fue el hecho de que la profesora del aula estaba constantemente en el aula por si surgía alguna duda o algún problema. Esta inseguridad fue disminuyendo a medida que iban pasando los grupos y ya tenía idea de cómo trabajar la ficha".

- "Me parece un Proyecto muy beneficioso, tanto para los alumnos como los docentes, ya que a los alumnos les ayuda y motiva en el aprendizaje y a los docentes les facilita un poco su labor, enriqueciendo así la educación en general. También me parece muy enriquecedor que tengan diversos voluntarios, ya que cada persona es un mundo y creo que pueden aprender mucho de ello"

\section{Reflexiones de algunos alumnos del Centro sobre los grupos interactivos}

Los alumnos de $5^{\circ}$ de Primaria reflexionan sobre los valores que se fomentan a través de los grupos interactivos manifestando opiniones tales como:

- Aprendemos a trabajar en equipo y a aprender cosas juntos.

- Nos prestamos muchas cosas, ayudamos a los demás, somos mejores compañeros y nos respetamos.

- Nos relacionamos más entre nosotros y con otros adultos, además de nuestra profesora.

- Colaboramos todos juntos para ayudar a aquel que no lo entiende.

- Podemos dar nuestra opinión, nos escuchamos y todos la respetamos.

- He aumentado la confianza de los demás.

- Aprendemos muchas cosas de otros países de donde vienen nuestros compañeros. Intercambio y enriquecimiento cultural.

- Si alguna vez estamos enfadados con otro compañero/a, aprovechamos esos momentos para acercarnos a él/ella para solucionar el problema dialogando.

Un alumno de $6^{\circ}$ de Primaria lo expresa así: 
- "Los compañeros en un grupo interactivo son muy importantes ya que pueden ser un ejemplo a seguir. Con ellos aprendes compañerismo, a respetar a tus compañeros y si no has entendido algo ellos te pueden ayudar a que lo entiendas. Por eso, pienso, que el compañero en un grupo interactivo es esencial".

\section{Reflexión desde la vivencia personal de una tutora, secretaria del Centro y coordinadora del voluntariado}

- En primer lugar, considero que las Comunidades de Aprendizaje y el trabajar con voluntarios son la solución actual más adecuada dentro de la sociedad que estamos viviendo hoy en día, ya que parten de los principios de la sociedad del conocimiento: la educación inclusiva o abierta todos, la libertad de expresión, el diálogo, conocimiento compartido, las tecnologías... Así como la sociedad cambia continuamente, los centros deberían transformarse social y culturalmente para crear entornos que mejoren la convivencia y la participación de todos.

- En segundo lugar, quiero destacar que desde en este centro hemos realizado muchos proyectos interesantes y la satisfacción personal que siento ahora mismo es enorme. Siento que cada día aporto mi granito de arena para mejorar la calidad de la educación; para que mis alumnos sepan actuar en la sociedad que les ha tocado vivir poniendo en práctica todos los valores que aprendemos $y$ practicamos en el centro $y$ he podido comprobar que los aprendizajes han mejorado para todos los alumnos, no sólo para unos pocos. Intentar transformar las dificultades en posibilidades, es algo que ilusiona y anima, a pesar de los problemas que nos encontramos en el día a día.

\section{DISCUSIÓN}

Al finalizar este trabajo queremos subrayar de nuevo la importancia que tiene la coordinación, colaboración y retroalimentación entre los centros escolares y las Universidades, como formadoras de los futuros maestros. A través de pequeñas, pero significativas experiencias queda claro que la simbiosis entre estos dos ámbitos educativos es sumamente beneficiosa para todos y, en definitiva, para una sociedad del futuro, que tanto deseamos, dotada no sólo de buenos y significativos conocimientos, sino también de buenos y significativos modos de convivir con los demás. Somos conscientes de que procesos como éstos requieren esfuerzos y tiempos adicionales, pero ello no nos va a desanimar, y menos impedir soñar, pues los sueños son los que nos pueden permitir construir un futuro mejor, formando unos maestros realmente comprometidos con los tiempos que vivimos, conocedores de la realidad y creativos frente a los retos que la misma dinámica local, regional y mundial vaya exigiendo de todos nosotros.

\section{REFERENCIAS}

Aguado, T. Gil, I. y Mata, P. (2007). El enfoque intercultural en la formación del profesorado. Dilemas y propuestas. Madrid: UNED. 
Aiguadé, I. P., Saso, C. E., Gallart, M. S., \& Carol, M. R. V. (2003). Comunidades de aprendizaje: transformar la educación (Vol. 177). Graó.

Cabrera, F., Espín, J., Marín, M., y Rodríguez, M. (1999). La formación del profesorado en educación multicultural. Construir la escuela intercultural. Reflexiones y propuestas para trabajar la diversidad étnica y cultural, p. 75-80.

Foncillas, M. Petreñas, C. y Cifuentes, A. (2012). Grupos interactivos: mejora del aprendizaje de todo el alumnado y de la convivencia en los centros educativos. Organización y Gestión educativa, 2.

Goenechea, C. (2008) ¿Es la formación del profesorado la clave de la educación intercultural? Revista Española de Pedagogía, 239, 119- 136.

Merino, D. y Leiva, J. J. (2006-07) El docente ante la realidad intercultural: Análisis de sus actitudes para la comprensión de sus nuevas funciones. El Guiniguada, 15-16, 195-206. 